\title{
Early clinical outcomes of isolated low velocity gunshot radius fractures treated with closed reduction and locked intramedullary nailing
}

S Maqungo FC Ortho, NJ Kauta MBChB, R Dachs FC Ortho, G McCollum FC Ortho, M Held FC Ortho, S Roche FCS(SA), Ortho

Orthopaedic Trauma Service, Department of Orthopaedic Surgery, Groote Schuur Hospital, University of Cape Town

\author{
Correspondence: \\ Dr Sithombo Maqungo \\ 4 Granula Place \\ 7441 Sunset Beach \\ Tel: +27832341723 \\ E-mail: sithombo@msn.com
}

\begin{abstract}
Background: The treatment goals in diaphyseal radius fractures are to regain and maintain length and rotational alignment and stability. Open reduction and plating carries the inherent problems of soft tissue disruption and periosteal stripping. Intramedullary nailing offers advantages of minimally invasive surgery and minimal soft tissue trauma.

Aims: To investigate the outcomes of locked intramedullary nailing for isolated gunshot diaphyseal radius fractures in adults.

Methods: A cross-sectional review of prospectively collected clinical and radiological data was performed. All adult patients with isolated gunshot radius fractures treated with closed reduction and locked intramedullary nailing between 2009 and 2013 were reviewed. Twenty-two nails were inserted in 22 patients, all males with a mean age of 28.9 years (range 19-40). All surgeries were performed between 2 and 12 days after injury (average 4 days). Follow-up was for an average of 11 weeks (range 8-24).

Results: All fractures united with the index procedure. Mean time to union was 10 weeks (range 8-24). Using the Anderson classification, the clinical outcome was excellent in 14 patients (64\%), satisfactory in six (27\%) and unsatisfactory in two $(9 \%)$.

Conclusion: Closed reduction and intramedullary nail fixation of gunshot radius fractures shows promising results without significant complications.
\end{abstract}

Key words: gunshot radius fractures, closed reduction, intramedullary nail fixation

http:/ / dx.doi.org/10.17159/2309-8309/2016/v15n2a3

\section{Introduction}

Diaphyseal forearm fractures in adults are treated with open reduction and plate osteosynthesis with consistently good and reproducible functional outcomes. However, this method requires more soft tissue dissection and periosteal stripping to achieve an anatomical reduction. ${ }^{1}$ The fracture comminution and soft tissue injury seen in low velocity gunshot injuries make the technique of open reduction and plate fixation less attractive for these fractures. Closed reduction intramedullary nail fixation offers all the advantages of minimally invasive surgery with regard to preservation of biology at the fracture site. ${ }^{2}$
We hypothesised that with the new generation forearm nail designs allowing proximal metaphyseal fit and distal locking screws, closed reduction and locked intramedullary nail fixation of isolated gunshot radius fractures would produce good clinical and functional results. The aim of our study was to assess early clinical outcomes of closed reduction and locked intramedullary fixation of low velocity gunshot radius fractures.

To our knowledge this is the first study in the English literature to report on isolated gunshot radius fractures in adults treated with an intramedullary nail. 


\section{Methods}

Prospectively collected clinical and radiological data on all patients who presented with an isolated midshaft or proximal third low velocity gunshot fracture of the radius between 2009 and 2013 were reviewed. Distal radial metaphyseal injuries were excluded from the study as these are not amenable to intramedullary nailing and are treated with a wrist-spanning external fixator in our unit. Ethics clearance was obtained from our institution's Ethics Committee and informed consent was obtained from all patients.

All patients received anti-tetanus toxoid and intramuscular penicillin injections on presentation. The wounds were covered in a sterile dressing and immobilised in a cast splint while awaiting definitive surgery. Low velocity gunshot wounds in the upper limb are not treated as open fractures in our unit so the surgery is not performed as an emergency.

The surgery was performed by different surgeons at our orthopaedic trauma unit using a standardised technique on all patients. Pre-operatively, all patients received intravenous cephazolin or clindamycin and this was continued for a further three doses post-operatively. We used the Acumed (Acumed, Hillsboro, Oregon) radius rod in all patients. All surgeries were performed under general anaesthesia and/or brachial plexus block. Patients were positioned supine with the affected arm surgically prepped on a radiolucent arm table. For the entry point a $1 \mathrm{~cm}$ longitudinal incision is made just ulnar to Lister's tubercle between the third and fourth extensor compartments. Under fluoroscopy a starter awl is used to breach the cortex starting $5 \mathrm{~mm}$ proximal to the articular surface. The smallest reamer is then advanced in a retrograde manner up to the fracture site, closed reduction is performed and the reamer advanced into the proximal radius. Reamers are sequentially inserted in appropriate increments to allow the insertion of a rod of desired length and diameter.

The fluted radius rod comes anatomically pre-bent to recreate the radial bow and it is left and right specific. The titanium composition allows further patient-specific contouring. We screen the unaffected radius in the operating room to estimate each patient's appropriate radial bow. The selected rod is advanced from distal to proximal and the proximal blade tip of the nail is anchored in the proximal metaphysis of the radius. Fluoroscopy is used to confirm final placement and correct depth. Rotation of the proximal fragment is checked under fluoroscopy using the position of the biceps tuberosity as described by Evans. ${ }^{3}$ Lastly distal locking is performed using the jig provided in a dorsal to volar direction (Figure 1).

No bone grafting was performed. In this surgical technique the fracture site is not opened. The devitalised edges of the bullet entry and exit wounds were debrided but not sutured and were allowed to heal by secondary intention.
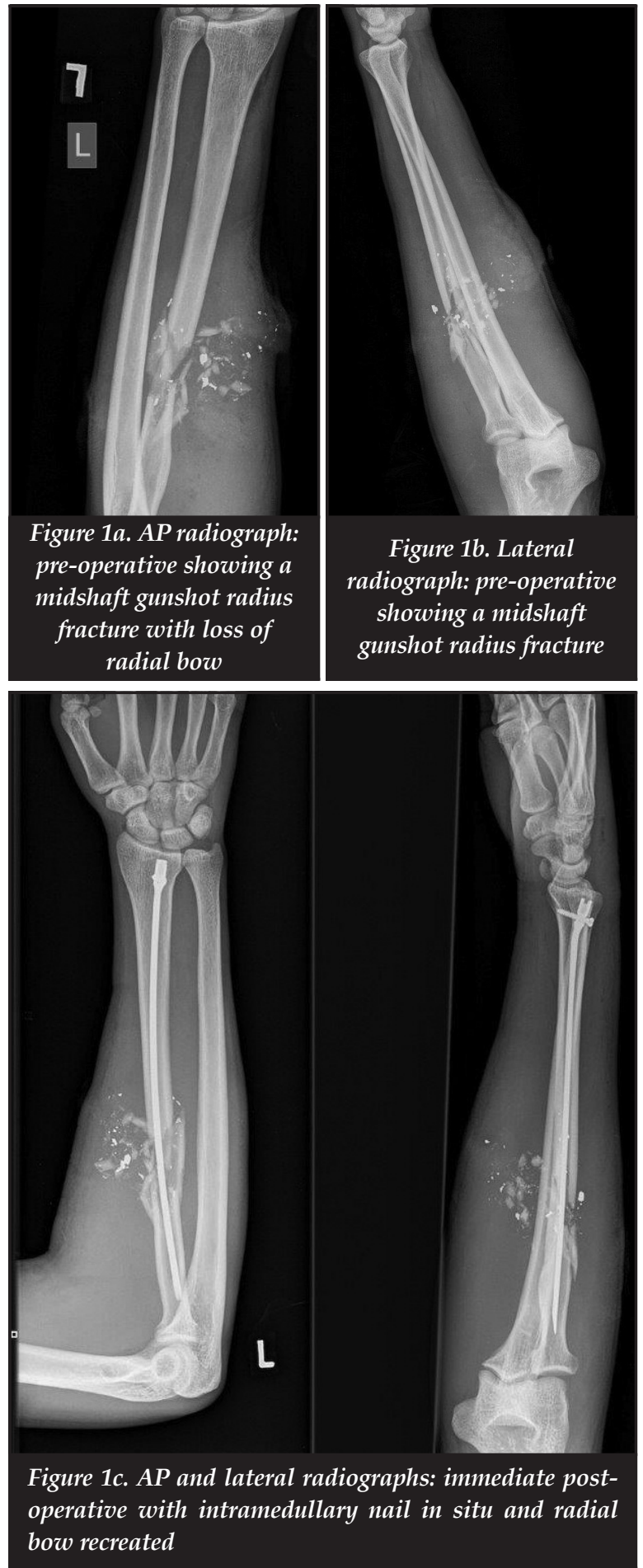

\begin{tabular}{|c|c|}
\hline Results & Criteria \\
\hline Excellent & $\begin{array}{l}\text { Fracture union } \\
\text { Loss of flexion/ extension of }<10^{\circ} \\
\text { Loss of pronation/supination of }<25 \%\end{array}$ \\
\hline Satisfactory & $\begin{array}{l}\text { Fracture union } \\
\text { Loss of flexion/extension of }<20^{\circ} \\
\text { Loss of pronation/supination of }<50 \%\end{array}$ \\
\hline Unsatisfactory & $\begin{array}{l}\text { Fracture union } \\
\text { Loss of flexion/extension of }>20^{\circ} \\
\text { Loss of pronation/supination of }>50 \%\end{array}$ \\
\hline Failure & $\begin{array}{l}\text { Fracture non-union } \\
\text { Unresolved chronic osteomyelitis }\end{array}$ \\
\hline
\end{tabular}


No attempt was made to irrigate the fracture site or remove retained bullets or shrapnel unless these were palpable subcutaneously. The limbs were not immobilised post-operatively, and unlimited range of movement physiotherapy was commenced on the day following surgery. Clinical and radiological follow-up was scheduled for weeks 2, 6, 10, 14, 18, 24 and 30 postoperatively. The range of forearm pronation and supination as well as wrist palmar flexion and dorsiflexion were recorded using a goniometer. The Anderson forearm fractures outcome classification (Table I) was used to determine the final outcome. ${ }^{4}$ We defined union clinically as lack of tenderness at the fracture site and radiographically as cross-trabeculation, the presence of bridging callus and obliteration of the fracture lines on three out of four cortices on two orthogonal radiographs. All radiographs were assessed by the corresponding author for union.

\section{Results}

Twenty-two radius rods were inserted in 22 patients. All were males with an average age of 29 years (range 1940). Two patients had pre-operative nerve injuries, a median nerve and the other a posterior interosseous nerve injury. There were no neurovascular complications recorded post-operatively. All procedures were performed between 2 and 12 days of the injury (average 4 days). Follow-up was for an average period of 11 weeks (range 8-24). All fractures united with the index procedure. The mean time to union was 10 weeks (range 8-24).

Fourteen patients $(64 \%)$ had an excellent outcome with a supination/pronation range of $75 \%$ or more of the normal side. Six $(27 \%)$ had a satisfactory outcome with supination/pronation range within $50 \%$ of the normal side. Two (9\%) had an unsatisfactory outcome with more than $20 \%$ loss of pronation/supination. These were the two patients who presented with pre-operative nerve injuries. Their nerve lesions did not recover and they were referred to our hand unit for definitive care.

One patient required nail removal at union due to its proximity to the radiocapitellar joint. This was not due to implant migration or fracture collapse, but was present in the immediate post-operative radiographs as the nail had been placed subchondrally. When comparing the post-operative radiographs with radiographs at union we observed no fracture displacement or loss of rotational alignment. There was no clinical or radiographic evidence that the nail had penetrated the joint. Orthogonal X-ray views were not helpful in terms of determining the exact location of the nail tip due to the concave nature of the proximal radial articular surface. Instead of doing a CT scan to confirm the exact location of the nail, we opted to remove the nail at union.

There were no superficial or deep infections recorded and there were no cases with radio-ulnar synostosis.

\section{Discussion}

The severity of soft tissue and bony injury caused by a bullet depends on the available kinetic energy, which is a function of its mass and velocity. ${ }^{5}$ Gunshot injuries are generally classified as low velocity (muzzle velocity of $<350 \mathrm{~m} / \mathrm{s}$ ), intermediate velocity $(350-500 \mathrm{~m} / \mathrm{s})$ and high velocity $(>600 \mathrm{~m} / \mathrm{s}){ }^{6}$ Literature suggests that low velocity gunshot fractures can be regarded as closed fractures and each fracture treated on its own merit even though the bullet is not sterilised on discharge. ${ }^{7.8}$

One constant feature of low velocity gunshot fractures is comminution and propagation. This particular feature renders compression plating impossible, and bridge plating the only viable plating technique. Bridge plating in the upper limb is typically performed by traditional open approaches. Gunshot fractures with a long segment of comminution require the use of long plates. The extensive soft tissue dissection may interfere with the biology of fracture healing and predispose the injury to post-operative infection or delayed union. Cadaveric and clinical studies have demonstrated that minimally invasive plate osteosynthesis can be safely performed for proximal humerus and humerus shaft fractures. ${ }^{910}$ Such studies are lacking for forearm fractures.

Restoring and maintaining the radial bow, proximal and distal radio-ulnar relationships and a stable fixation to allow early range of movement are the primary goals in the surgical treatment of forearm fractures.

Earlier intramedullary implants such as Rush rods and Steinman pins were neither axially nor rotationally stable. This led to the development of locked nails; for maximal stability these were locked both proximally and distally. Proximal locking (non-driving end) of these earlier implants posed a constant risk of injury to the posterior interosseous nerve and to avoid this they were locked via an open approach. The Acumed radius rod allows for distal locking (driving end) only and the non-driving end of the nail has a blade tip that anchors in the proximal radius metaphysis ensuring axial and rotational stability.

The available literature presents results of intramedullary fixation of both the radius and ulna without particular attention to the characteristics of the fractures treated nor their mechanism of injury.11-14 Some of the studies have used both open and closed reduction in their patients. All of them, however, have shown very positive results with regard to union rates and functional recovery.

Gelbart et al. presented a union rate of $92 \%$ in their prospective case series of low velocity gunshot forearm fractures treated with intramedullary nailing. ${ }^{15}$ Their study included both radius and ulna fractures which makes direct comparison to our study difficult.





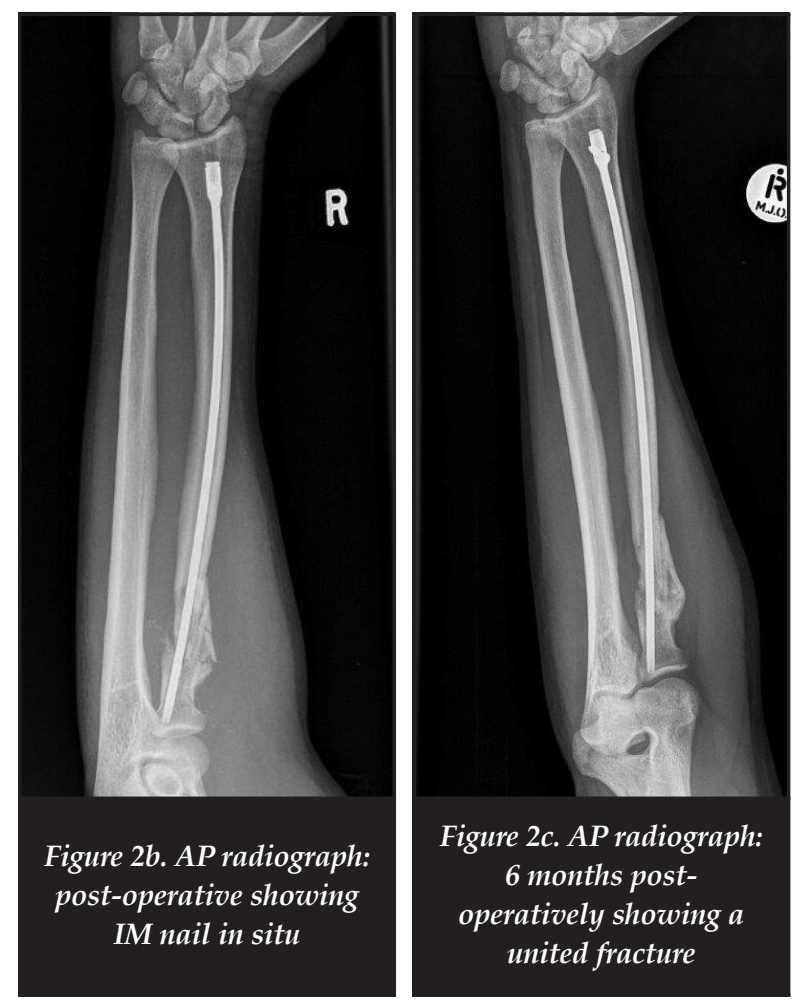

Our study is unique in that we reviewed patients with isolated radius fractures due to gunshot wounds. Saka et al. reported on 23 patients with isolated radius fractures treated with locked intramedullary nailing. ${ }^{16}$ Their patients were treated with a closed or mini open reduction and locked nailing and they achieved a mean time to union of 12 weeks. There were no gunshot injuries in their series. Our union rate of $100 \%$ and average time to union of 10 weeks compares very favourably with their results and other available studies that have used both plate fixation and intramedullary nailing.

We did not consider the two patients with a poor outcome as surgical complications. Both these patients had pre-operative nerve lesions that had not fully recovered at the time of union.

Intramedullary nailing offers the advantage of fixation of comminuted proximal third radius fractures where plate fixation would have been challenging and/or not technically possible (Figure 2).

A small sample size and the retrospective nature of this study represent its limitations.

\section{Conclusion}

Closed reduction and intramedullary nail fixation of gunshot radius fractures is a biologically and mechanically appealing fixation method for these complex diaphyseal fractures. These early clinical results are promising with respect to function and restoration of anatomy with no significant complications. Randomised control trials comparing the long-term outcomes of plate fixation and intramedullary nailing are required.

\section{Compliance with Ethics Guidelines}

Ethics clearance was obtained from our institution's Ethics Committee and informed consent was obtained from all patients.

S Maqungo, NJ Kauta, R Dachs, G McCollum, M Held and S Roche declare that no benefits in any form have been received or will be received from a commercial party related directly or indirectly to the subject of this article.

\section{References}

1. Chapman M, Gordon J, Zissimos A. Compression-plate fixation of acute fractures of the diaphysis of the radius and ulna. J Bone Joint Surg Am 1989;71(2):159-69.

2. Lil NA, Makkar DS, Aleem AA. Results of closed intramedullary nailing using Talwarkar square nails in adult forearm fractures. Malays Orthop J. 2012 Nov;6(3):7-12.

3. Evans EM. Fractures of the radius and ulna. J Bone Joint Surg Br 1951;33:548-61.

4. Anderson LD, Sisk D, Tooms RE, Park WI 3rd. Compressionplate fixation in acute diaphyseal fractures of the radius and ulna. J Bone Joint Surg Am 1975;57(3):287-97.

5. Bartlett CS, Helfet DL, Hausman MR, Strauss E. Ballistics and gunshot wounds: effects on musculoskeletal tissues. $J$ Am Acad Orthop Surg 2009;8(1):21-36.

6. Dickey RL, Barnes BC, Kearns RJ, Tullos HS. Efficacy of antibiotics in low-velocity gunshot fractures. J Orthop Trauma 1989;3(1):6-10.

7. Geissler WB, Teasedall RD, Tomasin JD, Hughes JL. Management of low-velocity gunshot induced fratcures. J Orthop Trauma. 1990;4(1):39-41.

8. Bowyer GW, Rossiter ND. Management of gunshot wounds to the limbs. J Bone Joint Surg Br. 1997 Nov;79(6):1031-36.

9. Apivatthakakul T, Arpornchayanon O, Bavornratanavech S. Minimally invasive plate osteosynthesis (MIPO) of the humeral shaft fracture. Is it possible? A cadaveric study and preliminary report. Injury. 2005 Apr;36(4):530-38.

10. Lau TW, Leung F, Chan CF, Chow SP. Minimally invasive plate osteosynthesis in the treatment of proximal humeral fracture. Int Orthop. 2007 Oct;31(5):657-64.

11. Gao H, Luo CF, Zhang CQ, Shi HP, Fan CY, Zen BF. Internal fixation of diaphyseal fractures of the forearm by interlocking intramedullary nail: short-term results in eighteen patients. J Orthop Trauma. 2005 Jul;19(6):384-91.

12. Weckbach $A$, Blattert TR, Weisser Ch. Interlocking nailing of forearm fractures. Arch Orthop Trauma Surg. 2006 Jul;126(5):309-15.

13. Lee YH, Lee SK, Chung MS, Baek GH, Gong HS, Kim KH. Interlocking contoured intramedullary nail fixation for selected diaphyseal fractures of the forearm in adults. J Bone Joint Surg Am. 2008 Sep;90(9):1891-98.

14. Saka G, Saglam N, Kurtulmuş T, Avcı CC, Akpinar F, Kovaci $\mathrm{H}$, Celik A. New interlocking intramedullary radius and ulna nails for treating forearm diaphyseal fractures in adults: a retrospective study. Injury. 2014 Jan;45 Suppl 1:S16-23.

15. Gelbart BR, Aden AA. Evaluation of intramedullary nailing in low velocity gunshot wounds of the radius and ulna. $S A$ Orthop Journal 2013;12(3):35-41.

16. Saka G, Saglam N, Kurtulmus T, Bakir U, Avci CC, Akpinar F, Alsaran A. Treatment of isolated diaphyseal fractures of the radius with an intramedullary nail in adults. Eur J Orthop Surg Traumatol. 2014 Oct;24(7):1085-93.

This article is also available online on the SAOA website (www.saoa.org.za) and the SciELO website (www.scielo.org.za). Follow the directions on the Contents page of this journal to access it. 